\title{
Notes on theta series for Niemeier lattices II
}

\author{
Shoyu Nagaoka
}

\begin{abstract}
Following [3], we study some congruence properties satisfied by the theta series associated with Niemeier lattices.
\end{abstract}

\section{Introduction}

This is a continuation of [3, where some congruence relations that a Siegel theta series $\vartheta_{\mathcal{L}}^{(n)}$ satisfies for a Niemeier lattice $\mathcal{L}$ were studied. The beginning of this study was the discovery of the congruence relation

$$
\Theta\left(\vartheta_{\omega}^{(2)}\right) \equiv 0 \quad(\bmod 23)
$$

where $\Theta$ is the theta operator and $\omega$ is the Leech lattice (for the definition, see [3, §2]). In [3, it was shown that this relation is a consequence of the fact that $\vartheta_{\omega}^{(2)}$ is congruent to the theta series for binary quadratic forms with discriminant -23 ([3, Theorem 3]). It should be noted that the prime number 23 is a prime factor of $|\operatorname{Aut}(\omega)|$ (the order of the automorphism group of the Leech lattice $\omega$ ).

In this study, we present another type of congruence relations, namely, mod 11 congruences between Siegel theta series for Niemeier lattices and theta series for quaternary quadratic forms.

Let $\left\{S_{1}, S_{2}, S_{3}\right\}$ be a set of representatives of the unimodular equivalence classes of the genus of the quaternary quadratic forms of discriminant $11^{2}$ with level 11 (for the explicit form, see $\S 3.1$ ).

The first result is as follows:

Theorem 1.1. The following congruence relations hold:

$$
\begin{aligned}
& \vartheta_{\alpha}^{(3)} \equiv \vartheta_{\kappa}^{(3)} \equiv \vartheta_{\psi}^{(3)} \equiv \vartheta_{S_{1}}^{(3)} \quad(\bmod 11) \\
& \vartheta_{\delta}^{(3)} \equiv \vartheta_{\iota}^{(3)} \equiv \vartheta_{\chi}^{(3)} \equiv \vartheta_{S_{2}}^{(3)} \quad(\bmod 11) \\
& \vartheta_{\epsilon}^{(3)} \equiv \vartheta_{\omega}^{(3)} \equiv \vartheta_{S_{3}}^{(3)}(\bmod 11) .
\end{aligned}
$$

where the Greek characters $\alpha, \kappa, \ldots$ denote the lattices labeled as in 2 , Table 16.1]. 
In [5], Ozeki obtained a large number of numerical data regarding the Fourier coefficients of Siegel theta series $\vartheta_{\omega}^{(n)}$ for the Leech lattice $\omega$. His calculations allow the generalization of a congruence relation that $\vartheta_{\omega}^{(4)}$ satisfies.

Theorem 1.2. We obtain

$$
\vartheta_{\omega}^{(4)} \equiv \vartheta_{S_{3}}^{(4)} \quad(\bmod 11) .
$$

In particular, the congruence relation

$$
\Theta\left(\vartheta_{\omega}^{(4)}\right) \equiv 0 \quad(\bmod 11)
$$

holds. Namely, $\vartheta_{\omega}^{(4)}$ is in the mod 11 kernel of the theta operator $\Theta$.

In the final section, we study some congruence relations that the theta series $\vartheta_{\omega}^{(n)}$ satisfies.

\section{Preliminaries}

\section{$2.1 \quad$ Notation}

The present notation primarily follows from 3 .

Let $\Gamma_{n}:=S p_{n}(\mathbb{Z})$ be the Siegel modular group of degree $n$, and let $\mathbb{H}_{n}$ be the Siegel upper half-space of degree $n$. For a subgroup $\Gamma \subset \Gamma_{n}$, we denote by $M_{k}(\Gamma)$ the $\mathbb{C}$-vector space of all Siegel modular forms of weight $k$ for $\Gamma$.

Any $F(Z)$ in $M_{k}\left(\Gamma_{n}\right)$ has a Fourier expansion of the form

$$
F(Z)=\sum_{0 \leq T \in \operatorname{Sym}_{n}^{*}(\mathbb{Z})} a(F ; T) q^{T}, \quad q^{T}:=\exp (2 \pi \sqrt{-1} \operatorname{tr}(T Z)), Z \in \mathbb{H}_{n},
$$

where

$$
\operatorname{Sym}_{n}^{*}(\mathbb{Z}):=\left\{T=\left(t_{i j}\right) \in \operatorname{Sym}_{n}(\mathbb{Q}) \mid t_{i i}, 2 t_{i j} \in \mathbb{Z}\right\} .
$$

For a subring $R$ in $\mathbb{C}$, let $M_{k}\left(\Gamma_{n}\right)_{R} \subset M_{k}\left(\Gamma_{n}\right)$ denote the $R$-module of all modular forms whose Fourier coefficients lie in $R$. As explained in $[3$, we may consider $F \in M_{k}\left(\Gamma_{n}\right)_{R}$ to be an element of the formal power series ring

$$
F \in \sum a(F ; T) q^{T} \in R\left[q_{i j}, q_{i j}^{-1}\right] \llbracket q_{11}, \ldots, q_{n n} \rrbracket
$$

when we write $q_{i j}:=\exp \left(2 \pi \sqrt{-1} z_{i j}\right)$ (cf. [3, §2.2]).

For a prime number $p$, we denote by $\mathbb{Z}_{(p)}$ the ring of $p$-integral rational numbers. For two elements

$$
F_{m}=\sum a\left(F_{m} ; T\right) q^{T} \in \mathbb{Z}_{(p)}\left[q_{i j}, q_{i j}^{-1}\right] \llbracket q_{11}, \ldots, q_{n n} \rrbracket \quad(m=1,2),
$$

we write $F_{1} \equiv F_{2}(\bmod p)$ if the congruence relation

$$
a\left(F_{1} ; T\right) \equiv a\left(F_{2} ; T\right) \quad(\bmod p)
$$


is satisfied for all $T$ with $0 \leq T \in S y m_{n}^{*}(\mathbb{Z})$.

We associate with $F=\sum a(F ; T) q^{T} \in M_{k}\left(\Gamma_{n}\right)$ the formal power series

$$
\Theta(F):=\sum a(F ; T) \cdot \operatorname{det}(T) q^{T} \in \mathbb{C}\left[q_{i j}, q_{i j}^{-1}\right] \llbracket q_{11}, \ldots, q_{n n} \rrbracket .
$$

It should be noted that $\Theta(F)$ is not necessarily a modular form. The operator $\Theta$ is called the theta operator (cf. [3, §2.4]).

\subsection{Theta series for lattices and matrices}

For a positive definite integral lattice $\mathcal{L}$ of degree $m$, we write the Gram matrix as $S=S_{\mathcal{L}} \in \operatorname{Sym}_{m}(\mathbb{Z})$. We associate with it the theta series

$$
\vartheta_{\mathcal{L}}^{(n)}=\vartheta_{S}^{(n)}(Z):=\sum_{X \in M_{m \times n}(\mathbb{Z})} \exp \left(\pi \sqrt{-1} \operatorname{tr}(S[X] Z), Z \in \mathbb{H}_{n},\right.
$$

where $S[X]={ }^{t} X S X$. In particular

$$
\vartheta_{\mathcal{L}}^{(n)}=\vartheta_{S}^{(n)}(Z) \in M_{\frac{m}{2}}\left(\Gamma_{n}\right)_{\mathbb{Z}}
$$

if $\mathcal{L}$ is an even unimodular lattice of degree $m$.

We now quote the following result, which will be used in the sequel and is a special case of a theorem by Böcherer and Nagaoka.

Theorem 2.1. (Böcherer-Nagaoka [1, Theorem 4]) We assume that $p \geq n+3$ and $p \equiv 3(\bmod 4)$. Let $S \in 2 S y m_{4}^{*}(\mathbb{Z})$ be an even integral, positive definite symmetric matrix with det $S=p^{2}$ with level $p$. Then, $\vartheta_{S}^{(n)} \in M_{2}\left(\Gamma_{0}^{n}(p)\right)$, and there exists a modular form $G \in M_{p+1}\left(\Gamma_{n}\right)_{\mathbb{Z}_{(p)}}$ such that

$$
\vartheta_{S}^{(n)} \equiv G \quad(\bmod p) .
$$

Proof. We apply [1, Theorem 4], where

$$
f=\vartheta_{S}^{(n)} \in M_{2}\left(\Gamma_{0}^{n}(p)\right)^{0}, \quad g=G \in M_{p+1}\left(\Gamma_{n}\right)
$$

in the notation of [1].

\subsection{Sturm bounds for Siegel modular forms}

We introduce a result of Richter and Westerholt-Raum [6] concerning the socalled Sturm bound.

Theorem 2.2. (Richter-Westerholt-Raum [6]) We assume that $p$ is a prime number and $F=\sum a(F ; T) q^{T}$ is a modular form in $M_{k}\left(\Gamma_{n}\right)_{\mathbb{Z}_{(p)}}(n \geq 2)$. If

$$
a(F ; T) \equiv 0 \quad(\bmod p)
$$


for all $0 \leq T=\left(t_{i j}\right) \in \operatorname{Sym}_{n}^{*}(\mathbb{Z})$ with

$$
t_{i i} \leq\left(\frac{4}{3}\right)^{n} \frac{k}{16} \quad(i=1, \ldots, n)
$$

then

$$
a(F ; T) \equiv 0 \quad(\bmod p)
$$

for all $0 \leq T \in \operatorname{Sym}_{n}^{*}(\mathbb{Z})$.

\section{Congruence properties of theta series for Niemeier lattices}

In [3], some congruence properties of theta series for Niemeier lattices were obtained. In this section, we present more such properties.

\subsection{Quaternary quadratic forms of discriminant $11^{2}$}

For later use, we consider some quaternary quadratic forms.

It follows from Nipp's table [4] that the genus of even positive quaternary quadratic forms of discriminant $11^{2}$ with level 11 consists of three classes, and their representatives are given by

$$
S_{1}=\left(\begin{array}{llll}
2 & 0 & 1 & 0 \\
0 & 2 & 0 & 1 \\
1 & 0 & 6 & 0 \\
0 & 1 & 0 & 6
\end{array}\right), \quad S_{2}=\left(\begin{array}{llll}
2 & 1 & 1 & 1 \\
1 & 2 & 0 & 1 \\
1 & 0 & 8 & 4 \\
1 & 1 & 4 & 8
\end{array}\right), \quad S_{3}=\left(\begin{array}{llll}
4 & 2 & 1 & 1 \\
2 & 4 & 0 & 1 \\
1 & 0 & 4 & 2 \\
1 & 1 & 2 & 4
\end{array}\right)
$$

By definition, we see that

$$
\vartheta_{S_{i}}^{(n)} \in M_{2}\left(\Gamma_{0}^{n}(11)\right)_{\mathbb{Z}} \quad(i=1,2,3) .
$$

\subsection{Congruence properties of theta series for Niemeier lat- tices}

In this subsection we prove some congruence relations that theta series for Niemeier lattices satisfy, which is our main result. We introduce the symbols that denote Niemeier lattices before describing the results. In 2, Conway and Sloane correspond the Greek characters $\alpha, \ldots, \omega$ to Niemeier lattices in the order of their Coxeter number. (cf. [2, Table 16.1]). The last lattice $\omega$ is the Leech lattice.

Theorem 3.1. The following congruence relations hold:

(i) $\vartheta_{\alpha}^{(3)} \equiv \vartheta_{\kappa}^{(3)} \equiv \vartheta_{\psi}^{(3)} \equiv \vartheta_{S_{1}}^{(3)}(\bmod 11)$,

(ii) $\quad \vartheta_{\delta}^{(3)} \equiv \vartheta_{\iota}^{(3)} \equiv \vartheta_{\chi}^{(3)} \equiv \vartheta_{S_{2}}^{(3)}(\bmod 11)$,

(iii) $\quad \vartheta_{\epsilon}^{(3)} \equiv \vartheta_{\omega}^{(3)} \equiv \vartheta_{S_{3}}^{(3)}(\bmod 11)$. 
where $\alpha, \kappa, \cdots$ are Niemeier lattices and $S_{1}, S_{2}, S_{3}$ are the Gram matrices given in (3.1).

Proof. (i) Let $h_{\mathcal{L}}$ denote the Coxeter number of the Niemeier lattice $\mathcal{L}$. We use the fact that the congruence relation $h_{\mathcal{L}_{1}} \equiv h_{\mathcal{L}_{2}}(\bmod n)$ implies $\vartheta_{\mathcal{L}_{1}}^{(3)} \equiv \vartheta_{\mathcal{L}_{2}}^{(3)}$ $(\bmod n)$ (cf. [3, Corollary 4]). As $h_{\alpha}=46, h_{\kappa}=13$, and $h_{\psi}=2$, we have $h_{\alpha} \equiv h_{\kappa} \equiv h_{\psi} \equiv 2(\bmod 11)$. This implies

$$
\vartheta_{\alpha}^{(3)} \equiv \vartheta_{\kappa}^{(3)} \equiv \vartheta_{\psi}^{(3)} \quad(\bmod 11) .
$$

We will prove the congruence

$$
\vartheta_{\alpha}^{(3)} \equiv \vartheta_{S_{1}}^{(3)} \quad(\bmod 11) .
$$

To this end, we use the results for the Sturm bound. Specifically, we first replace $\vartheta_{S_{1}}^{(3)}$ with a modular form for $\Gamma_{3}$ in the sense of modulo 11.

We note that $11 \equiv 3(\bmod 11)$ and that the matrix $S_{1}$ satisfies $\operatorname{det} S_{1}=11^{2}$ with level 11. Therefore, we can apply Theorem 2.1 to $\vartheta_{S_{1}}^{(3)}$. Namely, there is a modular form $G_{1} \in M_{12}\left(\Gamma_{3}\right)_{\mathbb{Z}_{(11)}}$ such that

$$
\vartheta_{S_{1}}^{(3)} \equiv G_{1} \quad(\bmod 11)
$$

We obtain the following table (for the abbreviation $T=[a, b, c]$, see [3, (10)]:

Table 1: Fourier coefficients: Degree 2 case

\begin{tabular}{c||rr}
\hline$T$ & $a\left(\vartheta_{S_{1}}^{(2)} ; T\right)$ & $a\left(\vartheta_{\alpha}^{(2)} ; T\right)$ \\
\hline$[0,0,0]$ & 1 & 1 \\
{$[1,0,0]$} & 4 & 1104 \\
{$[1,1,1]$} & 0 & 97152 \\
{$[1,0,1]$} & 8 & 1022304
\end{tabular}

If we use Theorem 2.2, we obtain $\vartheta_{\alpha}^{(2)} \equiv G_{1} \equiv \vartheta_{S_{1}}^{(2)}(\bmod 11)$. This implies that

$$
a\left(\vartheta_{\alpha}^{(3)} ; T\right) \equiv a\left(\vartheta_{S_{1}}^{(3)} ; T\right) \quad(\bmod 11)
$$

holds for all $T \in \operatorname{Sym}_{3}^{*}(\mathbb{Z})$ with $\operatorname{rank} T<3$. Moreover, we obtain the following numerical data (for the abbreviation $T=[a, b, c, ; d, e, f]$, see [3. (7)]): 
Table 2: Fourier coefficients: Degree 3 case

\begin{tabular}{c||rr}
\hline$T$ & $a\left(\vartheta_{S_{1}}^{(3)} ; T\right)$ & $a\left(\vartheta_{\alpha}^{(3)} ; T\right)$ \\
\hline$[1,1,1 ; 1,1,1]$ & 0 & 4177536 \\
{$[1,1,1 ; 0,0,1]$} & 0 & 81607680 \\
{$[1,1,1 ; 0,0,0]$} & 0 & 781393536
\end{tabular}

By using Theorem 2.2 again, we obtain

$$
a\left(\vartheta_{\alpha}^{(3)} ; T\right) \equiv a\left(\vartheta_{S_{1}}^{(3)} ; T\right) \quad(\bmod 11)
$$

for all $0 \leq T \in \operatorname{Sym}_{3}^{*}(\mathbb{Z})$. This completes the proof of (i).

The proofs of (ii) and (iii) are similar to that of (i). Concerning the Coxeter numbers, we have the following numerical data:

Table 3: Coxeter numbers

\begin{tabular}{c||rrr|rr} 
lattice & $\delta$ & $\iota$ & $\chi$ & $\epsilon$ & $\omega$ \\
\hline Coxeter number & 25 & 14 & 3 & 22 & 0
\end{tabular}

These imply that

$$
\vartheta_{\delta}^{(3)} \equiv \vartheta_{\iota}^{(3)} \equiv \vartheta_{\chi}^{(3)}(\bmod 11), \quad \vartheta_{\epsilon}^{(3)} \equiv \vartheta_{\omega}^{(3)} \quad(\bmod 11) .
$$

Thus, the proof is reduced to showing that

$$
\vartheta_{\delta}^{(3)} \equiv \vartheta_{S_{2}}^{(3)} \quad(\bmod 11), \quad \vartheta_{\omega}^{(3)} \equiv \vartheta_{S_{3}}^{(3)} \quad(\bmod 11),
$$

which can be obtained from the following tables:

Table 4: Fourier coefficients: Degree 2 case (Continued)

\begin{tabular}{c||rr|rr}
\hline$T$ & $a\left(\vartheta_{S_{2}}^{(2)} ; T\right)$ & $a\left(\vartheta_{\delta}^{(2)} ; T\right)$ & $a\left(\vartheta_{S_{3}}^{(2)} ; T\right)$ & $a\left(\vartheta_{\omega}^{(2)} ; T\right)$ \\
\hline$[0,0,0]$ & 1 & 1 & 1 & 1 \\
{$[1,0,0]$} & 6 & 600 & 0 & 0 \\
{$[1,1,1]$} & 12 & 27600 & 0 & 0 \\
{$[1,0,1]$} & 0 & 303600 & 0 & 0
\end{tabular}


Table 5: Fourier coefficients: Degree 3 case (Continued)

\begin{tabular}{c||rr|rr}
\hline$T$ & $a\left(\vartheta_{S_{2}}^{(3)} ; T\right)$ & $a\left(\vartheta_{\delta}^{(3)} ; T\right)$ & $a\left(\vartheta_{S_{3}}^{(3)} ; T\right)$ & $a\left(\vartheta_{\omega}^{(3)} ; T\right)$ \\
\hline$[1,1,1 ; 1,1,1]$ & 0 & 607200 & 0 & 0 \\
{$[1,1,1 ; 0,0,1]$} & 0 & 12751200 & 0 & 0 \\
{$[1,1,1 ; 0,0,0]$} & 0 & 127512000 & 0 & 0
\end{tabular}

This completes the proof of (ii) and (iii).

\section{Theta series for Leech lattice}

In this section, we will show that the congruence relations described in the previous section are extended to the case of the Leech lattice.

In [5], Ozeki provided a large number of data for the Fourier coefficients of theta series for the Leech lattice. His numerical data enable us to extend the congruence relation to the case of Leech lattice.

Theorem 4.1. Let $\omega$ be the Leech lattice and $S_{3}$ the matrix given in (3.1). Then, the following congruence relation holds:

$$
\vartheta_{\omega}^{(4)} \equiv \vartheta_{S_{3}}^{(4)} \quad(\bmod 11) .
$$

In particular,

$$
\Theta\left(\vartheta_{\omega}^{(4)}\right) \equiv 0 \quad(\bmod 11),
$$

where $\Theta$ is the theta operator defined in $\S$ 2.1.

Proof. We consider a modular form $G_{3} \in M_{12}\left(\Gamma_{4}\right)_{\mathbb{Z}_{(11)}}$ such that

$$
\vartheta_{S_{3}}^{(4)} \equiv G_{3} \quad(\bmod 11)
$$

as in the case of degree 3 . The congruence relation that we should prove is

$$
a\left(\vartheta_{\omega}^{(4)} ; T\right) \equiv a\left(\vartheta_{S_{3}} ; T\right)\left(\equiv a\left(G_{3} ; T\right)\right) \quad(\bmod 11)
$$

for all $0 \leq T \in \operatorname{Sym}_{4}^{*}(\mathbb{Z})$. As $\vartheta_{\omega}^{(3)} \equiv \vartheta_{S_{3}}^{(3)}(\bmod 11)$, it suffices to prove (4.1) for $T$ with $T>0$. Moreover, by considering the result for the Sturm bound (Theorem 2.2), it follows that (4.1) should be shown for $T$ satisfying

$$
0<T=\left(t_{i j}\right) \in \operatorname{Sym}_{4}^{*}(\mathbb{Z}) \text { with } t_{i i} \leq 2 \quad(i=1,2,3,4)
$$

If any of $t_{11}, \ldots, t_{44}$ is 1 under the condition (4.2) on $T$, we have $a\left(\vartheta_{\omega}^{(4)} ; T\right)=0$ because the lattice $\omega$ has no vectors of length 2. Moreover, from the identity

$$
a\left(\vartheta_{S_{3}}^{(4)} ; T\right)=\sharp\left\{G \mid S_{3}[G]=2 T\right\}
$$


and $\operatorname{det} S_{3}=11^{2}$, we see that

$$
a\left(\vartheta_{S_{3}}^{(4)} ; T\right)=0
$$

for $T$ whose discriminant satisfies $d_{T}<11^{2}$. Consequently, it suffices to prove (4.1) for $0<T=\left(t_{i j}\right) \in \operatorname{Sym}_{4}^{*}(\mathbb{Z})$ with

$$
t_{11}=t_{22}=t_{33}=t_{44}=2 .
$$

If we consider Table 6 in $\S 5$, we see that the target congruence relation (4.1) should be proved only for $T$ with $d_{T}=11^{2}$. In the case $d_{T}=11^{2}$, we have $a\left(\vartheta_{S_{3}}^{(4)} ; T\right)=\left|\operatorname{Aut}\left(S_{3}\right)\right|=24$ (cf. [4]). Consequently, we obtain

$$
a\left(\vartheta_{\omega}^{(4)} ; T\right)=12599323656192000 \equiv 24=a\left(\vartheta_{S_{4}}^{(4)} ; T\right) \quad(\bmod 11)
$$

for $T$ with $d_{T}=11^{2}$. This completes the proof of (4.1). The congruence relation $\Theta\left(\vartheta_{\omega}^{(4)}\right) \equiv 0(\bmod 11)$ is a consequence of $\vartheta_{\omega}^{(4)} \equiv \vartheta_{S_{3}}^{(4)}(\bmod 11)$.

\section{Fourier coefficients of theta series for Leech lattice}

\subsection{Ozeki's calculation}

In this subsection, we present a table concerning the Fourier coefficients of theta series for the Leech lattice calculated by Ozeki.

Table 6: Fourier coefficients of degree 4 theta series for Leech lattice by Ozeki [5. Table 5] : factorized.

\begin{tabular}{rll}
\hline$d_{T}$ & $T$ & $a\left(\vartheta_{\omega}^{(4)} ; T\right)$ \\
\hline 64 & $(2,2,2,2,0,0,0,2,2,2)$ & $2^{8} \cdot 3^{8} \cdot 5^{3} \cdot 7^{2} \cdot 11 \cdot 13 \cdot 23$ \\
80 & $(2,2,2,2,2,0,0,2,0,2)$ & $2^{11} \cdot 3^{8} \cdot 5^{3} \cdot 7^{2} \cdot 11 \cdot 13 \cdot 23$ \\
81 & $(2,2,2,2,1,1,1,1,2,2)$ & $2^{19} \cdot 3^{3} \cdot 5^{3} \cdot 7^{2} \cdot 11 \cdot 13 \cdot 23$ \\
84 & $(2,2,2,2,1,0,0,2,2,2)$ & $2^{16} \cdot 3^{7} \cdot 5^{3} \cdot 7 \cdot 11 \cdot 13 \cdot 23$ \\
96 & $(2,2,2,2,2,1,-1,0,0,2)$ & $2^{15} \cdot 3^{7} \cdot 5^{3} \cdot 7^{2} \cdot 11 \cdot 13 \cdot 23$ \\
105 & $(2,2,2,2,2,1,0,0,1,2)$ & $2^{19} \cdot 3^{7} \cdot 5^{3} \cdot 7 \cdot 11 \cdot 13 \cdot 23$ \\
108 & $(2,2,2,2,2,1,-1,-1,1,-1)$ & $2^{19} \cdot 3^{4} \cdot 5^{4} \cdot 7^{2} \cdot 11 \cdot 13 \cdot 23$ \\
112 & $(2,2,2,2,2,1,0,2,0,0)$ & $2^{16} \cdot 3^{8} \cdot 5^{4} \cdot 7 \cdot 11 \cdot 13 \cdot 23$ \\
116 & $(2,2,2,2,2,1,0,0,2,0)$ & $2^{16} \cdot 3^{8} \cdot 5^{3} \cdot 7^{2} \cdot 11 \cdot 13 \cdot 23$ \\
120 & $(2,2,2,2,1,1,1,2,2,0)$ & $2^{18} \cdot 3^{7} \cdot 5^{3} \cdot 7^{2} \cdot 11 \cdot 13 \cdot 23$ \\
121 & $(2,2,2,2,2,1,0,1,1,2)$ & $2^{20} \cdot 3^{8} \cdot 5^{3} \cdot 7^{2} \cdot 13 \cdot 23$
\end{tabular}


Table 7: (Continued)

\begin{tabular}{rll}
\hline$d_{T}$ & $T$ & $a\left(\vartheta_{\omega}^{(4)} ; T\right)$ \\
\hline 125 & $(2,2,2,2,1,1,-1,-1,1,1)$ & $2^{20} \cdot 3^{9} \cdot 5 \cdot 7^{2} \cdot 11 \cdot 13 \cdot 23$ \\
128 & $(2,2,2,2,0,0,0,2,2,0)$ & $2^{10} \cdot 3^{9} \cdot 5^{4} \cdot 7^{2} \cdot 11^{2} \cdot 13 \cdot 23$ \\
129 & $(2,2,2,2,1,1,1,1,2,2)$ & $2^{19} \cdot 3^{7} \cdot 5^{3} \cdot 7^{2} \cdot 11 \cdot 13 \cdot 23$ \\
132 & $(2,2,2,2,2,1,-1,0,0,1)$ & $2^{17} \cdot 3^{7} \cdot 5^{4} \cdot 7^{2} \cdot 11 \cdot 13 \cdot 23$ \\
140 & $(2,2,2,2,1,1,-1,0,0,2)$ & $2^{19} \cdot 3^{8} \cdot 5^{4} \cdot 7 \cdot 11 \cdot 13 \cdot 23$ \\
144 & $(2,2,2,2,2,1,-1,0,0,0)$ & $2^{16} \cdot 3^{7} \cdot 5^{3} \cdot 7^{2} \cdot 11 \cdot 13 \cdot 23^{2}$ \\
144 & $(2,2,2,2,2,0,0,0,0,2)$ & $2^{12} \cdot 3^{7} \cdot 5^{3} \cdot 7^{2} \cdot 11 \cdot 13 \cdot 23 \cdot 373$ \\
145 & $(2,2,2,2,2,1,0,-1,-1,1)$ & $2^{19} \cdot 3^{8} \cdot 5^{3} \cdot 7^{2} \cdot 11 \cdot 13 \cdot 23$ \\
153 & $(2,2,2,2,1,1,0,1,1,2)$ & $2^{19} \cdot 3^{7} \cdot 5^{4} \cdot 7^{2} \cdot 11 \cdot 13 \cdot 23$ \\
156 & $(2,2,2,2,1,1,1,2,0,0)$ & $2^{20} \cdot 3^{8} \cdot 5^{3} \cdot 7^{2} \cdot 11 \cdot 13 \cdot 23$ \\
160 & $(2,2,2,2,1,1,-1,1,-1,0)$ & $2^{15} \cdot 3^{8} \cdot 5^{3} \cdot 7^{2} \cdot 11 \cdot 13 \cdot 23 \cdot 41$ \\
160 & $(2,2,2,2,1,1,0,2,0,0)$ & $2^{18} \cdot 3^{8} \cdot 5^{4} \cdot 7^{2} \cdot 11 \cdot 13 \cdot 23$
\end{tabular}

Here, the following abbreviation was used:

$$
T=\left(t_{i j}\right)=:\left(t_{11}, t_{22}, t_{33}, t_{44}, t_{12}, t_{13}, t_{23}, t_{14}, t_{24}, t_{34}\right) .
$$

\subsection{Observation}

Herein, we provide some congruences that can be obtained by calculations analogous to those by Ozeki.

Ozeki [5] calculated the value $a\left(\vartheta_{\omega}^{(n)} ; T\right)$ for various degrees $n$ [5, Table 4,5,6]. We list the congruence relations expected from his tables.

$$
\begin{array}{ll}
\text { - } & \vartheta_{\omega}^{(n)} \equiv 1 \quad(\bmod 7) \\
\text { - } & \Theta\left(\vartheta_{\omega}^{(4)}\right) \equiv \Theta\left(\vartheta_{\omega}^{(5)}\right) \equiv 0 \quad\left(\bmod 7^{2}\right) .
\end{array}
$$

\section{References}

[1] S. Böcherer and S. Nagaoka, On Siegel modular forms of level $p$ and their properties mod $p$. manuscr. math. 132, 501-515(2010)

[2] J.H. Conway and N.J.A. Sloane, Sphere Packings, Lattices and Groups, 3rd edn. Springer, New York(1999)

[3] S. Nagaoka and S. Takemori, Notes on theta series for Niemeier lattices, The Ramanujan J. 42, 385-400(2017)

[4] G.L. Nipp, Quaternary Quadratic Forms, Computer Generated Tables, Springer, New York(1991) 
[5] M. Ozeki, Siegel theta series of various degrees for the Leech lattice, Kyushu J. Math. 68, 53-91(2014)

[6] O.K. Richter and M. Westerholt-Raum, Sturm bounds for Siegel modular forms, Research in Number Theory, 1-8(2015)

\author{
Shoyu Nagaoka \\ Department of Mathematics \\ Kindai University \\ Higashi-Osaka \\ Osaka $577-8502$ \\ Japan
}

